\title{
CERTAIN VARIETIES OF RESOLVING SETS OF A GRAPH
}

\author{
B. Sooryanarayana ${ }^{1}$, Suma A.S. ${ }^{2}$, Chandrakala S.B. $^{3}$ \\ ${ }^{1}$ Department of Mathematics, Dr. Ambedkar Institute of Technology, \\ Mallathalli, Bengaluru, INDIA, Pin 560 056, dr_bsnrao@dr-ait.org \\ ${ }^{2}$ Department of Mathematics, REVA University, Yelahanka, Bengaluru, \\ INDIA 560 064, suma.ts6@gmail.com \\ ${ }^{3}$ Department of Mathematics, Nitte Meenakshi Institute of Technology, \\ Yelahanka, Bengaluru, INDIA 560 064, chandrakalasb14@gmail.com
}

\begin{abstract}
Let $G=(V, E)$ be a simple connected graph. For each ordered subset $S=\left\{s_{1}, s_{2}, \ldots, s_{k}\right\}$ of $V$ and a vertex $u \in V$, we associate a vector $\Gamma(u / S)=$ $\left(d\left(u, s_{1}\right), d\left(u, s_{2}\right), \ldots, d\left(u, s_{k}\right)\right)$ with respect to $S$, where $d(u, v)$ denote the distance between $u$ and $v$ in $G$. A subset $S$ is called a resolving set of $G$ if $\Gamma(u / S) \neq$ $\Gamma(v / S)$ for all $u, v \in V-S$. The purpose of this paper is to introduce various types of $r$-sets and compute minimum cardinality of such sets. Using the superhereditaryness of resolving sets, it is shown that, for most of the graphs, the powerfull resolving and simple resolving numbers are identical. However, the foul resolving number is constant for cycles, paths and wheels, and the maximal resolving number is proportional to the order.
\end{abstract}

Key words and Phrases: Simple resolving sets, Metric dimensions, Landmarks, Powerful resolving sets, Maximal resolving sets, Foul resolving sets.

\section{INTRODUCTION}

All the graphs considered in this paper are connected, simple, undirected and finite. The distance between two vertices $a$ and $b$ of a graph $G$ is the length of a shortest path between them and is denoted by $d_{G}(a, b)$ or simply $d(a, b)$. The number of edges incident with a vertex $v$ of $G$ is the degree of the vertex $v$ in $G$ and is denoted by $\operatorname{deg}_{G}(v)$ or simply $\operatorname{deg}(v)$. A subset $S$ of a vertex set of $G$ is called a resolving set ( $r$-set) of $G$ if for each pair $u, v$ of vertices of $G$ there exist a vertex $w$ in $S$ with the property that $d(v, w) \neq d(u, w)$. The vertex $w$ is then called a resolving vertex for the pair $u, v$, or we say $w$ resolves $u$ and $v$. A resolving set $S$ is said to be a minimal resolving set of $G$ if none of its proper subsets are resolving set of $G$. The metric dimension of $G$, denoted by $\beta(G)$, is the minimum cardinality

2020 Mathematics Subject Classification: 05C20

Received: 08-10-2019, accepted: 25-01-2021. 
of a minimal resolving set of $G$. A resolving set with minimum cardinality is called a metric basis. The concept of metric dimension was introduced by F. Harary and R. A. Melter [5] and independently by P.J. Slater [10] under the term locating set. For more works on metric dimension, we refer $[3,4,6,8,9,11,12,7,14]$. The resolving property and dominating property of a network are super hereditary in nature and both are essential for unique identification of the nodes in the network for various purposes. The complement of a minimum dominating set is also a dominating set. Hence various classes of dominating sets are studied together with their complements. This is not the case of resolving sets, here the complement of a resolving set need not be a resolving set. For example every resolving set of a complete graph $K_{m}$ should include at least $m-1$ vertices of it. Hence, complement of a resolving set of $K_{m}$ can not be a resolving set for all $m \geq 3$.

In 2018, an attempt is made by B.Sooryanarayana and Suma A.S in [13] to study a special class of dominating set namely, the neighborhood set along with its complements and resolving property. In this paper, we extend the results of [13] by excluding the restriction of neighborhood property, so as to hold for more classes of resolving sets.

Throughout this paper $P_{m}$ denotes a path on $m$ vertices with the vertex set $V=\left\{v_{i}: 1 \leq i \leq m\right\}$ and the edge set $E=\left\{v_{i} v_{i+1}: 1 \leq i \leq m-1\right\}$, $C_{m}$ denotes a cycle on $m$ vertices with the vertex set $V=\left\{v_{i}: 1 \leq i \leq m\right\}$ and the edge set $E=\left\{v_{i} v_{i+1}: 1 \leq i \leq m-1\right\} \bigcup\left\{v_{1} v_{m}\right\}, K_{m}$ denotes the complete graph on $m$ vertices with the vertex set $V=\left\{v_{i}: 1 \leq i \leq m\right\}$ and the edge set $E=\left\{v_{i} v_{j}: i<j, 1 \leq i, j \leq m\right\}$ and $W_{1, m}$ denotes wheel graph on $m+1$ vertices with vertex set $V=\left\{v_{i}: 1 \leq i \leq m\right\} \bigcup\left\{v_{0}\right\}$ and edge set $E=\left\{v_{0} v_{i}: 1 \leq i \leq m\right\} \bigcup\left\{v_{i} v_{i+1}: 1 \leq i \leq m-1\right\} \bigcup\left\{v_{1} v_{m}\right\}$. The terms not defined here may be found in $[1,4]$.

\section{Relationship Between $p$-Sets And $p^{*}$-Sets}

A property $\mathcal{P}$ of a graph $G(V, E)$ is a class of subsets of $V$ and is called superhereditary if $S \in \mathcal{P}$ and $S \subseteq H$, then $H \in \mathcal{P}$. Equivalently, if $S \notin \mathcal{P}$ and $H \subseteq S$ implies that $H \notin \mathcal{P}$. For each $S \in \mathcal{P}$, the complement of $S$ in $V$ is $\bar{S}=V-S$. A property $\mathcal{P}^{*}$ is a subset of $\mathcal{P}$ such that for each $S \in \mathcal{P}^{*}$ we have $\bar{S} \in \mathcal{P}$. Clearly $\bar{S} \in \mathcal{P}^{*}$ and $\mathcal{P}^{*}$ need not be super-hereditary. A property $\hat{\mathcal{P}}$ is a subset of $\mathcal{P}$ such that for each $S \in \hat{\mathcal{P}}$ its complement $\bar{S} \notin \mathcal{P}$. Further, $\overline{\mathcal{P}}$ is a property of $G$ such that for each $S \in \overline{\mathcal{P}}$ neither $S \in \mathcal{P}$ nor its complement $\bar{S} \in \mathcal{P}$. An element of a property $\mathcal{P}$ is called a $p$-set if no proper subset of it is a member of $\mathcal{P}$. The minimum cardinality of a $p$-set are denoted by $l_{p}(G)$. Similarly, $l_{p^{*}}(G), l_{\hat{p}}(G)$ and $l_{\bar{p}}(G)$ are defined.

Theorem 2.1. Let $\mathcal{P}$ be a super-hereditary property of a graph $G$. Then either $l_{p}(G)=l_{p^{*}}(G)$ or $\mathcal{P}^{*}=\emptyset$. 
Proof. Let $S \in \mathcal{P}$ be a $p$-set of minimum cardinality and $P^{*} \neq \emptyset$. Let $S^{*} \in \mathcal{P}^{*}$ be an $p^{*}$-set with minimum cardinality in $G$. Since $S^{*} \in \mathcal{P}^{*} \subseteq \mathcal{P}$, we have $l_{p}(G)=|S| \leq$ $\left|S^{*}\right|$. On the other hand, if $\left|S^{*}\right|>|S|$, then for every $p$-set in $\mathcal{P}$ its complement is not in $\mathcal{P}$. Therefore, complement of each $p$-set of $G$ requires at least one more element from the $p$-set to make its complement a $p$-set, that is $\left|\overline{S^{*}}\right| \geq|\bar{S}|+1$ for every $p$-set $S$. But then, as $S^{*}$ is in $\mathcal{P}$ and $S$ is a $p$-set of minimum cardinality, $\left|S^{*}\right|>|S|$ and hence $\left|\overline{S^{*}}\right|<|\bar{S}| \leq\left|\overline{S^{*}}\right|-1$, a contradiction.

\section{Resolving Property of A Graph}

The resolving property $\mathcal{R}$ of a graph $G$, denoted by $\mathcal{R}(G)$, is super-hereditary. Each element of $\mathcal{R}(G)$ is a resolving set of $G$ and is also called simple resolving set. An $r$-set is a minimal element of $\mathcal{R}(G)$. The minimum cardinality of an $r$-set are the lower metric dimension of $G$ and is denoted by $l_{r}(G)$. Similarly, each element of $\mathcal{R}^{*}, \hat{\mathcal{R}}$ and $\overline{\mathcal{R}}$ are respectively called powerful resolving set, maximal resolving set and foul resolving set.

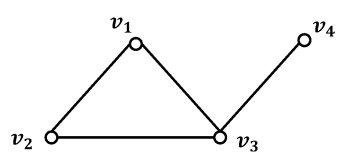

FIGURE 1. Illustration to resolving property of a graph

Let us consider a graph $G$ of Figure 1 , Let $\mathcal{R}(G)=\left\{\left\{v_{1}, v_{2}\right\},\left\{v_{1}, v_{3}\right\},\left\{v_{1}, v_{4}\right\}\right.$, $\left.\left\{v_{2}, v_{3}\right\},\left\{v_{2}, v_{4}\right\},\left\{v_{1}, v_{2}, v_{3}\right\},\left\{v_{1}, v_{2}, v_{4}\right\},\left\{v_{2}, v_{3}, v_{4}\right\},\left\{v_{1}, v_{3}, v_{4}\right\}, V(G)\right\}$ among these elements of $\mathcal{R}(G)$ minimal $r$-sets of $G$ are $\left\{v_{1}, v_{2}\right\},\left\{v_{1}, v_{3}\right\},\left\{v_{1}, v_{4}\right\},\left\{v_{2}, v_{3}\right\}$, $\left\{v_{2}, v_{4}\right\}$ with cardinality 2. Hence, $l_{r}(G)=2$. Let $\mathcal{R}^{*}(G)=\left\{\left\{v_{1}, v_{3}\right\},\left\{v_{2}, v_{4}\right\}\right.$, $\left.\left\{v_{1}, v_{4}\right\},\left\{v_{2}, v_{3}\right\}\right\}$ and these sets are minimal sets with cardinality 2. Hence, $l_{r}^{*}(G)=2$. Let $\hat{\mathcal{R}}(G)=\left\{\left\{v_{1}, v_{2}\right\},\left\{v_{1}, v_{2}, v_{3}\right\},\left\{v_{1}, v_{2}, v_{4}\right\},\left\{v_{2}, v_{3}, v_{4}\right\},\left\{v_{1}, v_{3}, v_{4}\right\}\right\}$ among these elements the set $\left\{v_{1}, v_{2}\right\}$ is a minimal set with cardinality 2 . Hence, $l_{\hat{r}}(G)=2$. The set $\overline{\mathcal{R}}(G)=\emptyset$ due to the fact that either $S$ or $\bar{S}$ is an $r$-set.

Remark 3.1. If $G$ is a non-trivial graph of order $m$ and $S$ is any subset of vertices of $G$, then
i) $S \in \mathcal{R}(G)$, if $|S| \geq m-1$.
ii) $1 \leq l_{r}(G) \leq|V(G)|-1$.
iii) $|S|<m-1$, if $S \notin R(G)$.

Theorem 3.2 ([6]). Let $l_{r}(G)=2$ and $\{a, b\} \in \mathcal{R}(G)$. If $P$ is a shortest path between $a$ and $b$ in $G$, then the following are true:

1. $P$ is unique.

2. $\operatorname{deg}_{G}(a) \leq 3$ and $\operatorname{deg}_{G}(b) \leq 3$.

3. $\operatorname{deg}_{G}(v) \leq 5$, for every $v \in V(P)$. 


\section{4. $r$-Sets and Dimensions of A Path}

Remark 4.1. From the definition of an $r$-set, it is clear that every 2-element subset of vertices of a path $P_{m}$ is in $\mathcal{R}\left(P_{m}\right)$ for every $m \geq 2$. In fact, if $S=\{a, b\}$ and $u, v$ be arbitrary vertices of $P_{m}$ such that $d(u, a)=d(v, a)$, then a is the central vertex of the uv-path in $P_{m}$, but then exactly one of the paths, ub-path or vb-path, in $P_{m}$ contains the vertex $a$ and hence $d(u, b) \neq d(v, b)$.

Remark 4.2. A singleton set $\{v\} \in \mathcal{R}(G)$ if and only if $G \cong P_{m}$ and $v$ is an end vertex of $P_{m}$ for some $m \in Z^{+}$.

Observation 4.3. Every member of $\mathcal{R}\left(P_{m}\right)$ contains a pendent vertex of $P_{m}$ whenever $1 \leq m \leq 3$.

Proposition 4.4. For any positive integer $m \geq 2, l_{r}\left(P_{m}\right)=1=l_{r^{*}}\left(P_{m}\right)$.

Proof. An immediate consequence of Theorem 2.1 and Remark 4.2.

Theorem 4.5. For any positive integer $m, l_{\hat{r}}\left(P_{m}\right)=\left\{\begin{array}{lll}m & \text { for } & m=1,2 . \\ m-1 & \text { for } & \text { otherwise. }\end{array}\right.$

Proof. Let $S \in \hat{\mathcal{R}}\left(P_{m}\right)$ and $l_{\hat{r}}\left(P_{m}\right)=|S|$. Then $S \in \mathcal{R}\left(P_{m}\right)$ and $\bar{S} \notin \mathcal{R}\left(P_{m}\right)$. Hence by Remark 4.1 and Remark $4.2,|\bar{S}| \nsupseteq 2$ and $\bar{S}$ not contains any end vertex of $P_{m}$. Therefore, $|S| \geq m-1$ if $m \geq 3$, and $|S|=m$ if $m=1,2$. But then, by Remark $3.1,|S|=m-1$ if $m \geq 3$, and $|S|=m$ if $m=1,2$ (Since $S$ is a minimal $r$-set).

Remark 4.6. For each $m \in Z^{+}, \overline{\mathcal{R}}\left(P_{m}\right)=\emptyset$ due to the fact that either $S \in \mathcal{R}\left(P_{m}\right)$ or $\bar{S} \in \mathcal{R}\left(P_{m}\right)$, for every $S \subseteq V\left(P_{m}\right)$.

\section{5. $r$-Sets And Dimensions of A Cycle}

Theorem 5.1. Let $S$ be an $i$-element subset of vertices of a cycle $C_{m}$ and $3 \leq i \leq$ $m$. Then $S$ is in $\mathcal{R}\left(C_{m}\right)$.

Proof. Let $S$ be a subset of the vertices of $C_{m}$ with cardinality at least 3 . Let $a, b, c \in S$ and $x, y$ be any two vertices of cycle $C_{m}$ for $m \geq 3$. If possible, let $d(a, x)=d(a, y)$ and $d(b, x)=d(b, y)$. Then $a$ and $b$ lie in distinct $x y$-paths in $C_{m}$ and $m$ is even. In case if $c$ lies between $a$ and $x$, then $d(c, x)<d(c, y)$ and hence $c$ resolves the pair $x, y$. Similarly, other cases follows by symmetry.

Remark 5.2. Let $m \geq 3$ and $v_{0}, v_{1}, \ldots, v_{m-1}$ be the vertices of $C_{m}$ in order. Then the subset $\left\{v_{i}, v_{i \oplus_{m} 1}\right\}$ is in $\mathcal{R}\left(C_{m}\right)$ for every $i \in \mathbb{Z}_{m}$.

Theorem 5.3 ([6]). For every integer $m \geq 3, l_{r}\left(C_{m}\right)=2$.

Theorem 5.4. For each integer $m \geq 3, l_{r}\left(C_{m}\right)=l_{r^{*}}\left(C_{m}\right)=2$ if $m \neq 3, l_{r}\left(C_{3}\right)=$ 2 , and $\mathcal{R}^{*}\left(C_{3}\right)=\emptyset$. 
Proof. The case $m \geq 4$ follows from Theorem 2.1, Remark 5.2 and Theorem 5.3. Further, for $m=3$, by Theorem $5.3,|S| \geq 2$ for each $S \in \mathcal{R}\left(C_{3}\right)$ and hence $|\bar{S}| \leq 1$. So, $\bar{S} \notin \mathcal{R}\left(C_{3}\right)$. Hence $l_{r}\left(C_{3}\right)=2$ and $\mathcal{R}^{*}\left(C_{3}\right)=\emptyset$.

Lemma 5.5. The set $\{a, b\}$ is in $\mathcal{R}\left(C_{m}\right)$ if and only if either (i) $m$ is odd or (ii) $m$ is even and $d_{C_{m}}(a, b) \neq \frac{m}{2}$.

Proof. Let $P$ be a shortest path between $a$ and $b$ in $C_{m}$.

Let $\{a, b\} \in \mathcal{R}\left(C_{m}\right)$. If $m$ is odd then nothing to prove. We now suppose that $m$ is even. If possible, let $d_{C_{m}}(a, b)=\frac{m}{2}$. Then $\bar{P}=\left\langle V\left(C_{m}\right)-V(P)\right\rangle$ is also a shortest path between $a$ and $b$ in $C_{m}$ other than $P$, a contradiction to the fact that $\{a, b\}$ is in $\mathcal{R}\left(C_{m}\right)$ (by Theorem 3.2).

Conversely, let $x$ and $y$ be any two arbitrary vertices of $C_{m}$. If $d(a, x)=$ $d(a, y)$ and $d(b, x)=d(b, y)$, then both $x$ and $y$ cannot be in a common short path between $a$ and $b$. Let $x \in V(P)$ and $y \in V(\bar{P})$. But then, $m=|V(P)|+$ $|V(\bar{P})|=d(a, x)+d(x, b)+d(a, y)+d(y, b)=2(a, x)+2 d(x, b)=2|V(P)|=$ even and $|V(P)|=d(a, b)=\frac{m}{2}$, a contradiction.

Theorem 5.6. For any integer $m \geq 3$,

$$
l_{\hat{r}}\left(C_{m}\right)= \begin{cases}m-2 & \text { if } m \text { is even and } m \neq 4 . \\ m-1 & \text { otherwise. }\end{cases}
$$

Proof. Let $S \in \hat{\mathcal{R}}\left(C_{m}\right)$ be such that $l_{\hat{r}}\left(C_{m}\right)=|S|$. Then $S \in \mathcal{R}\left(C_{m}\right)$ and $\bar{S} \notin$ $\mathcal{R}\left(C_{m}\right)$. If $m$ is odd then by Remark $4.2,|S| \geq 2$ (since $\left.G \neq P_{m}\right)$ and $|\bar{S}|<2$ (since $\bar{S} \notin \mathcal{R}\left(C_{m}\right)$ and $\mathcal{R}$ is super-hereditary). Thus, $|S|=m-1$. If $m$ is even, $|\bar{S}| \leq 2$ (since $\{a, b\} \notin \mathcal{R}\left(C_{m}\right)$ with $d(a, b)=\frac{m}{2}$ by Lemma 5.5). Hence $|S|=m-2$ if $m \neq 4$ with two antipodal vertices in $\bar{S}$. Finally, when $m=4, S$ should contain two adjacent vertices to become an $r$-set but then $\bar{S}$ resolves (by Lemma 5.5) and hence $|S| \geq 3$. Hence $|S|=3$ (by Remark 3.1) if $m=4$.

For $m=3$ or $m \geq 5$ and a subset $S$ of the vertices of the cycle $C_{m}$, by Remark 5.2 and the super-hereditary property of $\mathcal{R}(G)$, it follows that either $S \in$ $\mathcal{R}\left(C_{m}\right)$ or $\bar{S} \in \mathcal{R}\left(C_{m}\right)$. Hence $\overline{\mathcal{R}}\left(C_{m}\right)=\emptyset$ for $m=3$ or $m \geq 5$. However, for a subset $S$ consisting of any two antipodal vertices of $C_{4}$, neither $S \in \mathcal{R}\left(C_{m}\right)$ nor $\bar{S} \in \mathcal{R}\left(C_{m}\right)$. Thus we have the following theorem.

Theorem 5.7. For an integer $m \geq 3, l_{\bar{r}}\left(C_{4}\right)=2$ and $\overline{\mathcal{R}}\left(C_{m}\right)=\emptyset$ if $m \neq 4$.

\section{6. $r$-Sets and Dimensions of A Complete Graph}

Theorem 6.1. For any positive integer $m, l_{r}\left(K_{m}\right)= \begin{cases}1 & \text { for } m=1 . \\ m-1 & \text { otherwise. }\end{cases}$

Proof. Let $S \in \mathcal{R}\left(K_{m}\right)$ such that $l_{r}\left(K_{m}\right)=|S|$. Then for each $x, y \in V\left(K_{m}\right)-S$, $d(x, w)=d(y, w)=1$ for each $w \in S$. Hence $|\bar{S}| \leq 1$ implies that $|S| \geq m-1$ if $m \geq 2$ and $|S|=1$ if $m=1$ (since $S$ is non-empty). 
If $S \in \mathcal{R}^{*}\left(K_{m}\right)$, then from the proof of the above theorem, we see that $|S| \geq m-1$ and $|\bar{S}| \geq m-1$. Hence such a set $S$ exists (with $|S|=1$ ) if and only if $m=2$. We record this fact in the form of the following theorem.

Theorem 6.2. For any positive integer $m, l_{r^{*}}\left(K_{m}\right)=1$ if $m=2$, and $\mathcal{R}^{*}\left(K_{m}\right)=\emptyset$ if $m \neq 2$.

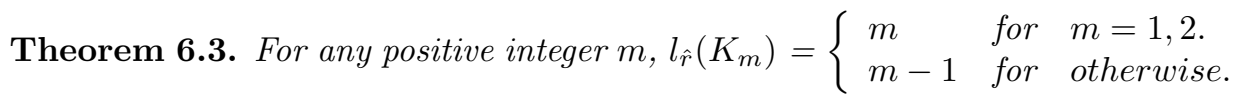

Proof. Let $S \in \hat{\mathcal{R}}\left(K_{m}\right)$ be such that $|S|=l_{\hat{r}}\left(K_{m}\right)$. Then $S \in \mathcal{R}\left(K_{m}\right)$ and $\bar{S} \notin$ $\mathcal{R}\left(K_{m}\right)$. For the case $m \leq 2$, the result follows by Theorem 4.5. Let $m \geq 3$. Then $|S| \geq m-1$ (by Theorem 6.1) and $\{v\} \notin \mathcal{R}\left(K_{m}\right)$ for each $v \in V\left(K_{m}\right)$ (by Remark 4.2). Hence $|S|=m-1$.

Theorem 6.4. For any positive integer $m, l_{\bar{r}}\left(K_{m}\right)=2$ if $m \geq 4$, and $\overline{\mathcal{R}}\left(K_{m}\right)=\emptyset$ if $m \leq 3$.

Proof. Let $S \in \overline{\mathcal{R}}\left(K_{m}\right)$ be such that $|S|=l_{\bar{r}}\left(K_{m}\right)$. Then $S \notin \mathcal{R}\left(K_{m}\right)$ and $\bar{S} \notin$ $\mathcal{R}\left(K_{m}\right)$. So, by Remark 3.1, $2 \leq|S| \leq m-2$ and $2 \leq|\bar{S}| \leq m-2$. Hence, $m \geq 4$ and $l_{\bar{r}}\left(K_{m}\right)=2$.

\section{7. $r$-Sets And Dimensions of A Wheel}

Theorem 7.1 ([9]). If $W_{1, m}$ is the wheel graph for $m \geq 3$, then

(i) $l_{r}\left(W_{1,3}\right)=l_{r}\left(W_{1,6}\right)=3$.

(ii) $l_{r}\left(W_{1,4}\right)=l_{r}\left(W_{1,5}\right)=2$.

(iii) $l_{r}\left(W_{1, x+5 k}\right)=\left\{\begin{array}{lll}3+2 k, & \text { for } x=7 \text { or } 8 . \\ 4+2 k, & \text { for } x=9 \text { or } 10 \text { or } 11 .\end{array}\right.$

Theorem 7.2. For any positive integer $m \geq 3$,

$$
l_{r}\left(W_{1, m}\right)=\left\{\begin{array}{cll}
3 & \text { for } & m=3,6 \\
2 & \text { for } & m=4,5 \\
\left\lfloor\frac{2(m+1)}{5}\right\rfloor, & \text { for } & m \geq 7
\end{array}\right.
$$

Proof. The result follows from Theorem 7.1.

Definition 7.3. A Chinese fan graph $F(1, m)$ is the sum graph $K_{1}+P_{m}$. i.e it is a graph obtained by taking a vertex $v$ adding it to every vertex of the path $P_{m}$ by an edge.

Theorem 7.4 ([2]). For any integer $m, l_{r}(F(1, m))=\left\{\begin{array}{cll}1 & \text { if } & m=1 \\ 2 & \text { if } & m=2,3 \\ 3 & \text { if } & m=6 \\ \left\lfloor\frac{2 m+2}{5}\right\rfloor & \text { if } & \text { otherwise. }\end{array}\right.$ 
Definition 7.5. Let $G(V, E)$ be a graph and $S \subseteq V$. Then for any two vertices $u, v \in V$, a path between $u$ and $v$ is called an $\bar{S}$ path if each vertex of the path other than the end vertices are in $\bar{S}$.

Definition 7.6. If there exists an $\bar{S}$ path between $u, v \in S$, then we define the gap between $u$ and $v$ in $S$, denoted by $g_{S}(u, v)$, as the number of vertices of $\bar{S}$ in a shortest $\bar{S}$ path between $u$ and $v$.

For example, in the graph $G$ of Figure 2, the shortest $\bar{S}$ path between $a$ and $e$ is $a-c-d-e$ if $S=\{a, b, e\}$. Therefore $g_{\{a, b, e\}}(a, e)=2$. Similarly, $g_{\{a, d, e\}}(a, e)=1, g_{\{a, b, c\}}(a, b)=0, g_{\{a, d, f\}}(a, d)=1$, and $g_{\{a, b, d, e\}}(a, e)=3$. Further, $g_{\{a, b\}}(a, b)=d(a, b)-1=0$.

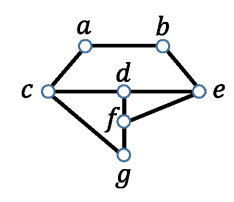

FiguRE 2. The graph $G$.

Lemma 7.7. For any integer $m \geq 3$ and a subset $S \subseteq V\left(W_{1, m}\right)$, if $g_{S \cup\left\{c_{0}\right\}}(a, b) \geq 2$ and $g_{S \cup\left\{c_{0}\right\}}(a, c) \geq 2$, for some $a, b, c \in S$ with $b \neq c$, then $S \notin \mathcal{R}\left(W_{1, m}\right)$.

Proof. Let $g_{S \cup\left\{c_{0}\right\}}(a, b) \geq 2$, for some $a, b \in S$. Then $a-v_{i}-v_{i+1}-\cdots-b$ is a shortest $\left.\overline{S \cup\left\{c_{0}\right.}\right\}$ path between $a$ and $b$ for some $i$. Further, if $g_{S \cup\left\{c_{0}\right\}}(a, c) \geq 2$ and $b \neq c$ then, there are two rim vertices $v_{j}, v_{j+1}$ in $\bar{S}$ with $i \neq j$, such that $a-v_{j}-v_{j+1}-\cdots-c$ is a shortest $\overline{S \cup\left\{c_{0}\right\}}$ path between $a$ and $c$. But then $d\left(a, v_{i}\right)=d\left(a, v_{j}\right)=1$ and $d\left(s, v_{i}\right)=d\left(s, v_{j}\right)=2$, for all $s \in S-\{a\}$. Therefore, none of the vertices in $S$ will resolve the pair $v_{i}, v_{j}$. Hence $S \notin \mathcal{R}\left(W_{1, m}\right)$.

Lemma 7.8. For any integer $m \geq 3$ and a subset $S \subseteq V\left(W_{1, m}\right)$, if $g_{S \cup\left\{c_{0}\right\}}(a, b) \geq 4$ for some $a, b \in S$, then $S \notin \mathcal{R}\left(W_{1, m}\right)$.

Proof. Let $g_{S \cup\left\{c_{0}\right\}}(a, b) \geq 4$, for some $a, b \in S$. Then $a=v_{i}-v_{i+1}-v_{i+2}-\ldots-$ $v_{i+k}=b$ is a shortest $\overline{S \cup\left\{c_{0}\right\}}$ path between $a$ and $b$ for some $i, k$ with $k \geq 6$. But then $d\left(s, v_{i+2}\right)=d\left(s, v_{i+3}\right)=2$ for all $s \in S$. Therefore, none of the vertices in $S$ will resolve the pair $v_{i+2}, v_{i+3}$. Hence $S \notin \mathcal{R}\left(W_{1, m}\right)$.

Lemma 7.9. For any integer $m \geq 3$ and a subset $S \subseteq V\left(W_{1, m}\right)$, if $g_{S \cup\left\{c_{0}\right\}}(a, b)=3$ and $g_{S \cup\left\{c_{0}\right\}}(c, d)=3$, for some $a, b, c, d \in S$ with $a \neq c$ then $S \notin \mathcal{R}\left(W_{1, m}\right)$.

Proof. Let $g_{S \cup\left\{c_{0}\right\}}(a, b)=3$ and $g_{S \cup\left\{c_{0}\right\}}(c, d)=3$, for some $a, b, c, d \in S$. Then $a=v_{i}-v_{i+1}-v_{i+2}-v_{i+3}-v_{i+4}=b$ and $c=v_{j}-v_{j+1}-v_{j+2}-v_{j+3}-v_{j+4}=d$, for some $i, j$ and $i \neq j$ are the shortest $\overline{S \cup\left\{c_{0}\right\}}$ path between the $a, b$ and $c, d$ respectively. But then, $d\left(s, v_{i+2}\right)=d\left(s, v_{j+2}\right)=2$ for all $s \in S$. Therefore, none of the vertices in $S$ will resolve the pair $v_{i+2}, v_{j+2}$. Hence $S \notin \mathcal{R}\left(W_{1, m}\right)$. 
Remark 7.10. For each $i, 1 \leq i \leq m$, it is easy to see that $\left\{v_{i}, v_{i+1}\right\} \in \mathcal{R}\left(W_{1, m}\right)$ for each $m=4,5$.

Theorem 7.11. A set $S$ with of cardinality at least three is in $\mathcal{R}\left(W_{1, m}\right)$ for any $m \geq 6$ if and only if the following hold.

(i) $g_{S \cup\left\{c_{0}\right\}}\left(v_{i}, v_{j}\right) \leq 3$ for every $v_{i}, v_{j} \in S$, and $g_{S \cup\left\{c_{0}\right\}}\left(v_{i}, v_{j}\right)=3$ for at most one pair $v_{i}, v_{j} \in S$.

(ii) $g_{S \cup\left\{c_{0}\right\}}\left(v_{i}, v_{i+3}\right)=2$ and $g_{S \cup\left\{c_{0}\right\}}\left(v_{i+k}, v_{i+k+3}\right) \geq 2$, for some $v_{i}, v_{i+3}, v_{i+k}$, $v_{i+k+3} \in S$ implies $4 \leq k \leq 5$.

Proof. Let $S \subseteq V\left(W_{1, m}\right)$ be satisfying the conditions (i) and (ii). Since $|S| \geq 3, S$ has a rim vertex. Without loss of generality we take rim vertex $v_{1} \in S$. If possible, let us assume the contrary that $S \notin \mathcal{R}\left(W_{1, m}\right)$. Then there exist $u, v \in V\left(W_{1, m}\right)-S$ such that

$$
d(u, w)=d(v, w) \quad \forall w \in S .
$$

We now prove the theorem by contradiction in different cases as follows.

Case 1: $d(u, w)=d(v, w)=1$ for every $w \in S$.

Subcase 1: $c_{0} \in\{u, v\}$.

In this case, from Equation 1, we get $1=d(u, w)=d(v, w)$ for all $w \in S$. So, in particular $d\left(v, v_{1}\right)=1$ implies that $v=v_{2}\left(\right.$ or $\left.v=v_{m}\right)$ and hence $m=3$, a contradiction.

Subcase 2: $c_{0} \notin\{u, v\}$.

In this case, from Equation 1, we get $1=d(u, w)=d(v, w)$ for all $w \in$ $S$. So, in particular $d\left(u, v_{1}\right)=d\left(v, v_{1}\right)=1$ implies that $u, v \in\left\{v_{2}, v_{m}\right\}$. But then, for any $i, 1<i<m$ if $v_{i} \in S$ then, $d\left(v_{2}, v_{i}\right)=d\left(v_{m}, v_{i}\right)=1$ (from Equation 1 with $w=v_{i}$ ) implies that $i=3$ and hence $m=4$, a contradiction.

Case 2: $d(u, w)=d(v, w)=2$, for some $w \in S$.

In this case, $c_{0} \notin\{u, v\}$. Therefore, $u=v_{i}$ and $v=v_{j}$ for some $1<i<$ $j \leq m$. But then, $v_{i-1}, v_{i+1}, v_{j-1}, v_{j+1} \notin S$. Let $P$ be a path between $u$ and $v$ in $C_{m}$ containing the vertex $v_{1}$.

Subcase 1: $V(\bar{P}) \cap S=\emptyset$.

In this case, either $v_{i-1}, v_{i}, v_{j}, v_{j+1} \in V(\bar{P})$ or $v_{i}, v_{i+1}, v_{j-1}, v_{j} \in$ $V(\bar{P})$. Hence, as there are no other vertices of $\bar{P}$ in $S$, for the vertices $a, b \in S$ that are nearer to the end vertices of $\bar{P}$, we get $g_{\{a, b\}}=$ $\left[\left|V\left(C_{m}\right)\right|-|V(P)|\right]+2 \geq 4$, a contradiction to condition (i).

Subcase 2: $V(\bar{P}) \cap S \neq \emptyset$.

Let $v_{k} \in V(\bar{P}) \cap S$. Let $a$ and $c$ be the vertices in $S \cap V(\bar{P})$ nearer to $v_{j}$ and $v_{i}$, respectively. Let $b$ and $d$ be the vertices in $S \cap V(P)$ be a vertex nearer to $v_{j}$ and $v_{i}$, respectively. Then, $g_{\{a, b\}}=$ [length of the path $P_{1}$ between $a$ and $b$ containing $\left.v_{j}\right]-1 \geq 3$ (since $v_{j-1}, v_{j}, v_{j+1} \in V\left(P_{1}\right) \cap$ $\bar{S})$ and $g_{\{c, d\}}=$ [length of the path $P_{2}$ between $c$ and $d$ containing $\left.v_{i}\right]-1 \geq 3$ (since $v_{i-1}, v_{i}, v_{i+1} \in V\left(P_{s}\right) \cap \bar{S}$ ), a contradiction to the condition (i). 
Converse part follows by Lemma 7.7, Lemma 7.8 and Lemma 7.9.

Theorem 7.12. For any positive integer $m \geq 4$

$$
l_{r^{*}}\left(W_{1, m}\right)=\left\{\begin{array}{cll}
3, & \text { for } & m=6 . \\
2, & \text { for } & m=4,5 \\
\left\lfloor\frac{2(m+1)}{5}\right\rfloor, & \text { for } & m \geq 7 .
\end{array}\right.
$$

Proof. In view of Theorem 2.1 and Theorem 7.2, it suffices to show that $\mathcal{R}^{*}\left(W_{1, m}\right) \neq$ $\emptyset$, for all $m \geq 4$.

If $m=4,5$, then the set $S=\left\{v_{1}, v_{2}\right\} \in \mathcal{R}\left(W_{1,4}\right)$ and $\bar{S} \in \mathcal{R}\left(W_{1,4}\right)$, So Also it is easy to see that the set $S=\left\{v_{1}, v_{2}, v_{3}\right\} \in \mathcal{R}^{*}\left(W_{1,6}\right)$. Let $m \geq 7$.

Case 1: $m \equiv 0(\bmod 5)$.

Let $S=\left\{v_{1}, v_{6}, v_{11}, \ldots, v_{m-4}\right\} \cup\left\{v_{4}, v_{9}, v_{14}, \ldots, v_{m-1}\right\}$.

Case 2: $m \equiv 1(\bmod 5)$.

Let $S=\left\{v_{1}, v_{6}, v_{11}, \ldots, v_{m-4}\right\} \cup\left\{v_{4}, v_{9}, v_{14}, \ldots, v_{m-6}, v_{m}\right\}$.

Case 3: $m \equiv 2(\bmod 5)$.

Let $S=\left\{v_{1}, v_{6}, v_{11}, \ldots, v_{m+1}\right\} \cup\left\{v_{4}, v_{9}, v_{14}, \ldots, v_{m-1}\right\}$.

Case 4: $m \equiv 3(\bmod 5)$.

Let $S=\left\{v_{1}, v_{6}, v_{11}, \ldots, v_{m-4}\right\} \cup\left\{v_{4}, v_{9}, v_{14}, \ldots, v_{m-6}, v_{m}, v_{m+2}\right\}$.

Case 5: $m \equiv 4(\bmod 5)$.

Let $S=\left\{v_{1}, v_{6}, v_{11}, \ldots, v_{m-4}\right\} \cup\left\{v_{4}, v_{9}, v_{14}, \ldots, v_{m-6}, v_{m-1}\right\}$.

In all the above cases the set $S$ as well as $\bar{S}$ satisfies all the conditions (i) and (ii) of Theorem 7.11. Hence $\bar{S} \in \mathcal{R}\left(W_{1, m}\right)$. Therefore, $\mathcal{R}^{*}\left(W_{1, m}\right) \neq \emptyset$.

Remark 7.13. If $m=3$, then the graph $W_{1,2} \cong K_{4}$ and hence by Theorem 6.2, $\mathcal{R}^{*}\left(W_{1, m}\right)=\emptyset$.

Theorem 7.14. For any integer $m \geq 3, l_{\bar{r}}\left(W_{1, m}\right)=\left\{\begin{array}{lll}2 & \text { for } & m=3,4 \\ 4 & \text { for } & m \geq 8 .\end{array}\right.$

Proof. Let $S \in \overline{\mathcal{R}}\left(W_{1, m}\right)$ be such that $|S|=l_{\bar{r}}\left(W_{1, m}\right)$. Then $S \notin \mathcal{R}\left(W_{1, m}\right)$ and $\bar{S} \notin$ $\mathcal{R}\left(W_{1, m}\right)$. If $m=3$, then $W_{1,3} \cong K_{4}$ and hence the result is true by Theorem 6.4. If $m=4$, then by Remark 3.1, we have $|\bar{S}| \leq 3$. So, $|S| \geq 2$. Further, the set $\left\{v_{1}, v_{3}\right\} \notin \mathcal{R}\left(W_{1,4}\right)$ and its complement $\left\{v_{2}, v_{0}, v_{4}\right\} \notin \mathcal{R}\left(W_{1,4}\right)$. Therefore, $l_{\bar{r}}\left(W_{1,4}\right)=\left|\left\{v_{1}, v_{3}\right\}\right|=2$.

Let us now consider the case $m \geq 8$. If $|S| \leq 3$, then $g_{\bar{S} \cup\left\{c_{0}\right\}}(a, b) \leq 3$, for all $a, b \in \bar{S}$ and hence $\bar{S} \in \mathcal{R}\left(W_{1, m}\right)$, a contradiction. Hence $l_{\bar{r}}\left(W_{1, m}\right)=|S| \geq 4$. Now, to prove the reverse inequality, consider the set $T=\left\{v_{2}, v_{3}, v_{4}, v_{5}\right\}$. For this set $T$, we see that $g_{T \cup\left\{c_{0}\right\}}\left(v_{2}, v_{5}\right) \geq 4$ and $g_{\bar{T} \cup\left\{c_{0}\right\}}\left(v_{1}, v_{6}\right) \geq 4$. So, by Theorem 7.11, $T \notin \mathcal{R}\left(W_{1, m}\right)$ and $\bar{T} \notin \mathcal{R}\left(W_{1, m}\right)$. Hence the set $T \in \overline{\mathcal{R}}\left(W_{1, m}\right)$. Thus, $l_{\bar{r}}\left(W_{1, m}\right) \leq|T|=4$.

Remark 7.15. For $5 \leq m \leq 7$ and for any subset $S$ of vertices of $W_{1, m}$ either the set $S$ or the set $\bar{S}$ satisfies the conditions (i) and (ii) of Theorem \%.11. Therefore, $\overline{\mathcal{R}}\left(W_{1, m}\right)=\emptyset$ for $5 \leq m \leq 7$. 
Lemma 7.16. For any integer $m \geq 9$, if $S \in \mathcal{R}\left(W_{1, m}\right)$ and $|S|=l_{r}\left(W_{1, m}\right)$, then $g_{\bar{S} \cup\left\{c_{0}\right\}}(a, b) \leq 2$ for all $a, b \in \bar{S}$.

Proof. Let $S \in \mathcal{R}\left(W_{1, m}\right)$. If possible let $g_{\bar{S} \cup\left\{c_{0}\right\}}(a, b) \geq 3$ some $a, b \in \bar{S}$. Then $\left\{v_{i}, v_{i+1}, v_{i+2}\right\} \subset S$ for some $1 \leq i \leq m-2$. But then, the set $S^{\prime}=S-\left\{v_{i+1}\right\}$ satisfies the condition (i) and (ii) of Theorem 7.11 (since, for all $c, d \in S^{\prime}$, we have $g_{S^{\prime} \cup\left\{c_{0}\right\}}(c, d)=1$ if $\{c, d\}=\left\{v_{i}, v_{i+2}\right\}, g_{S^{\prime} \cup\left\{c_{0}\right\}}(c, d)=g_{S \cup\left\{c_{0}\right\}}(c, d)$ otherwise, and $S$ satisfies conditions (i) and (ii)). Hence $S^{\prime} \in \mathcal{R}\left(W_{1, m}\right)$ with $\left|S^{\prime}\right|<|S|$, a contradiction to the fact that $|S|=l_{r}\left(W_{1, m}\right)$.

Lemma 7.17. For any integer $m \geq 9$, if $S \in \mathcal{R}\left(W_{1, m}\right)$ and $|S|=l_{r}\left(W_{1, m}\right)$, then $\bar{S} \in \mathcal{R}\left(W_{1, m}\right)$.

Proof. Let $S \in \mathcal{R}\left(W_{1, m}\right)$ and $|S|=l_{r}\left(W_{1, m}\right)$. If possible, let $\bar{S} \notin \mathcal{R}\left(W_{1, m}\right)$. Then, by Lemma 7.16 and Theorem 7.11, without loss of generality we get $\left\{v_{i-2}\right.$, $\left.v_{i-1}, v_{i+1}, v_{i+2}\right\} \subset S$ for some $v_{i} \in \bar{S}, 3 \leq i \leq m-2$. But then, the set $S^{\prime}=$ $\left[S \cup\left\{v_{i}\right\}\right]-\left\{v_{i-1}, v_{i+1}\right\}$ satisfies all the conditions of Theorem 7.11 (since, for all $c, d \in S^{\prime}$, we have $g_{S^{\prime} \cup\left\{c_{0}\right\}}(c, d)=1$ if $\{c, d\}=\left\{v_{i-2}, v_{i}\right\}$ or $\{c, d\}=\left\{v_{i}, v_{i+2}\right\}$, $g_{S^{\prime} \cup\left\{c_{0}\right\}}(c, d)=g_{S \cup\left\{c_{0}\right\}}(c, d)$ otherwise, and $S$ satisfies conditions (i) and (ii)). Hence $S^{\prime} \in \mathcal{R}\left(W_{1, m}\right)$ with $\left|S^{\prime}\right|<|S|$, a contradiction to the fact that $|S|=l_{r}\left(W_{1, m}\right)$

Lemma 7.18. Let $S \in \mathcal{R}\left(W_{1, m}\right)$ and $m \equiv 1(\bmod 5)$. Then if $g_{S \cup\left\{C_{0}\right\}}\left(v_{i-1}, v_{i+1}\right)=$ $g_{S \cup\left\{C_{0}\right\}}\left(v_{i+1}, v_{i+3}\right)=1$ for some $v_{i-1}, v_{i+1}, v_{i+3} \in S$, then $|S|>l_{r}\left(W_{1, m}\right)$.

Proof. Let $m=5 k+1$ and $k \geq 2$. Then, by Theorem 7.2 , we have $l_{r}\left(W_{1, m}\right)=2 k$. Let $S \in \mathcal{R}\left(W_{1, m}\right)$ be arbitrary. If $\left\{v_{j+1}, v_{j+2}, v_{j+3}\right\} \not \subset \bar{S}$ for any $v_{j}, v_{j+4} \in S$, then by condition (ii) of Theorem 7.11, we get $|S| \geq\left\lceil\frac{2 m}{5}\right\rceil=2 k+1$ (since for every five element subset of $V\left(W_{1, m}\right)$ has at least 2 elements in $S$ ). Hence the result holds in this case. Else if, $\left\{v_{j+1}, v_{j+2}, v_{j+3}\right\} \subset \bar{S}$ for some $v_{j}, v_{j+4} \in S$, then without loss of generality we can take $j=1$ and hence $v_{1}, v_{5} \in S$. But then, by condition (ii) of the Theorem 7.11, $\left|\left\{v_{k-1}, v_{k}, v_{k+1}, v_{k+2}, v_{k+3}\right\} \cap S\right| \geq 2$ for every $k \geq 3$. Now if $g_{S \cup\left\{C_{0}\right\}}\left(v_{i-1}, v_{i+1}\right)=g_{S \cup\left\{C_{0}\right\}}\left(v_{i+1}, v_{i+3}\right)=1$, for some $v_{i-1}, v_{i+1}, v_{i+3} \in S$, then $\left|\left\{v_{i-1}, v_{i}, v_{i+1}, v_{i+2}, v_{i+3},\right\} \cap S\right|=3$. Hence, we get $|T|=\sum_{i=1}^{(m-1) / 5}\left|T_{i}\right|>\frac{2(m-1)}{5}=2 k$, where $T_{i}=\left\{v_{5 i-3}, v_{5 i-2}, v_{5 i-1}, v_{5 i}, v_{5 i+1}\right\}$.

Lemma 7.19. For any integer $m \geq 9, l_{\hat{r}}\left(W_{1, m}\right) \geq l_{r}\left(W_{1, m}\right)+\xi$, where $\xi=2$ if $m \equiv 1(\bmod 5)$ and $\xi=1$ otherwise.

Proof. Let $S \in \hat{\mathcal{R}}\left(W_{1, m}\right)$ with $|S|=l_{\hat{r}}\left(W_{1, m}\right)$. Then $S \in \mathcal{R}\left(W_{1, m}\right)$ and $\bar{S} \notin$ $\mathcal{R}\left(W_{1, m}\right)$. So, by Lemma 7.17 it is suffices to prove the case $m \equiv 1$ under modulo 5. Let $m=5 k+1$ and $k \geq 2$. If possible, let $|S|=l_{r}\left(W_{1, m}\right)+1=\left\lfloor\frac{2 m+2}{5}\right\rfloor+1=$ $\left\lfloor\frac{2(5 k+1)+2}{5}\right\rfloor+1=2 k+1$. Since $\bar{S} \notin \mathcal{R}\left(W_{1, m}\right)$, by Theorem 7.11 , we have (i) $\left\{v_{i}, v_{i+1}, v_{i+2}, v_{i+3}\right\} \subset S$ or (ii) $\left\{v_{i}, v_{i+1}, v_{i+3}, v_{i+4}\right\} \subset S$ for some $v_{i+2} \notin S$ and $1 \leq i \leq m-4$ or (iii) $\left\{v_{i}, v_{i+1}, v_{i+3}, v_{i+k}, v_{i+k+1}, v_{i+k+2}\right\} \subset S$ for some $k>4$ and $5<i+k \leq m-2$.

Case 1: $\left\{v_{i}, v_{i+1}, v_{i+2}, v_{i+3}\right\} \subset S$. 
Consider the set $S^{\prime}=\left[S \cup\left\{v_{i+4}\right\}\right]-\left\{v_{i+1}, v_{i+3}\right\}$ (or $S^{\prime}=\left[S \cup\left\{v_{i-1}\right\}\right]-$ $\left\{v_{i+1}, v_{i+3}\right\}$ if $i+4>m$ ). The set $S^{\prime} \in \mathcal{R}\left(W_{1, m}\right)$ (since it $S \in \mathcal{R}\left(W_{1, m}\right)$ and no two element of $S^{\prime}$ will create a gap more than 2). Further, $g_{S^{\prime} \cup\left\{C_{0}\right\}}\left(v_{i}, v_{i+2}\right)=1=$ $g_{S^{\prime} \cup\left\{C_{0}\right\}}\left(v_{i+2}, v_{i+4}\right)$ and hence by Lemma 7.18 , we get $|S|-1 \geq\left|S^{\prime}\right|>l_{r}\left(W_{1, m}\right) \Rightarrow$ $|S| \geq l_{r}\left(W_{1, m}\right)+2$.

Case 2: $\left\{v_{i}, v_{i+1}, v_{i+3}, v_{i+4}\right\} \subset S$ for some $v_{i+2} \notin S$ and $1 \leq i \leq m-4$.

Consider the set $S^{\prime}=\left[S \cup\left\{v_{i+2}\right\}\right]-\left\{v_{i+1}, v_{i+3}\right\}$. The set $S^{\prime} \in \mathcal{R}\left(W_{1, m}\right)$ (since $S \in \mathcal{R}\left(W_{1, m}\right)$ and no two element of $S^{\prime}$ will create a gap more than 2). Further, $g_{S^{\prime} \cup\left\{C_{0}\right\}}\left(v_{i}, v_{i+2}\right)=1=g_{S^{\prime} \cup\left\{C_{0}\right\}}\left(v_{i+2}, v_{i+4}\right)$ and hence by Lemma 7.18, we get $|S|-1=\left|S^{\prime}\right|>l_{r}\left(W_{1, m}\right) \Rightarrow|S| \geq l_{r}\left(W_{1, m}\right)+2$.

Case 3: $\left\{v_{i}, v_{i+1}, v_{i+3}, v_{i+k}, v_{i+k+1}, v_{i+k+2}\right\} \subset S$ for some $k>4$ and $5<$ $i+k \leq m-2$.

Consider the set $S^{\prime}=S-\left\{v_{i+1}, v_{i+k+1}\right\}$. The set $S^{\prime} \in \mathcal{R}\left(W_{1, m}\right)$ (since $S \in \mathcal{R}\left(W_{1, m}\right)$ and no two element of $S^{\prime}$ will create a gap more than 2) and hence $|S|-2=\left|S^{\prime}\right| \geq l_{r}\left(W_{1, m}\right) \Rightarrow|S| \geq l_{r}\left(W_{1, m}\right)+2$.

Theorem 7.20. For any positive integer $m \geq 3$,

$$
l_{\hat{r}}\left(W_{1, m}\right)=\left\{\begin{array}{cl}
m, & \text { for } \quad m=3 . \\
\left\lfloor\frac{2(m+4)}{5}\right\rfloor, & \text { for } \quad m \geq 4 .
\end{array}\right.
$$

Proof. Let $S \in \hat{\mathcal{R}}\left(W_{1, m}\right)$ be such that $|S|=l_{\hat{r}}\left(W_{1, m}\right)$. Then $S \in \mathcal{R}\left(W_{1, m}\right)$ and $\bar{S} \notin \mathcal{R}\left(W_{1, m}\right)$. For $m=3$, the result holds by Theorem 6 .3. For the case $m=4,5$; if $S$ has only two adjacent rim vertices, then $\bar{S}$ contains two adjacent rim vertices. Hence, by Remark $7.10, \bar{S} \in \mathcal{R}\left(W_{1,4}\right)$, a contradiction. Or, if $m=4,5$ and $S$ has only two non-adjacent rim vertices $v_{i}, v_{i+2}$, then $S$ will not resolve the pair $c_{0}, v_{i+1} \in \bar{S}$, a contradiction to the fact that $S \in \mathcal{R}\left(W_{1, m}\right)$. Thus, $|S|=3$ for $m=$ 4,5 (Since $\left\{v_{1}, v_{2}, v_{3}\right\} \in \hat{\mathcal{R}}\left(W_{1,4}\right)$ and $\left.\left\{v_{1}, v_{2}, v_{4}\right\} \in \hat{\mathcal{R}}\left(W_{1,5}\right)\right)$. If $m=6$ and $|S|=2$, then only possible $S=\left\{v_{i}, v_{i+2}\right\}$ for some $1 \leq i \leq 4$ (follows by Theorem 7.12). But then, $S$ will not resolve the pair $c_{0}, v_{i} \in \bar{S}$, again a contradiction to the fact that $S \in \mathcal{R}\left(W_{1, m}\right)$. Further, any set $S$ containg any three rim elements of $W_{1,6}$ satisfies the conditions (i) and (ii) of Theorem 7.14 and hence $l_{\hat{r}}\left(W_{1,6}\right)=3$.

If $m=7,8$, then by Theorem 7.2 we have $|S| \geq 3$ (since $S \in \mathcal{R}\left(W_{1, m}\right)$ ). Further if $|S|=3$, then $\langle S\rangle$ is disconnected (else condition (i) of Theorem 7.8 fails) and hence $g_{\bar{S} \cup\left\{c_{0}\right\}}(a, b) \leq 2$ for all $a, b \in \bar{S}$. So, $\bar{S} \in \mathcal{R}\left(W_{1, m}\right)$ (Theorem 7.8), a contradiction. Therefore, $l_{\hat{r}}\left(W_{1, m}\right)=|S| \geq 4$. Further, for the subset $T=$ $\left\{v_{i}, v_{i+1}, v_{i+3}, v_{i+4}\right\}$ we see that $g_{T \cup\left\{c_{0}\right\}}\left(v_{i+1}, v_{i+3}\right)=1$ and $g_{T \cup\left\{c_{0}\right\}}\left(v_{i}, v_{i+4}\right) \geq 2$. So, the set $T \in \mathcal{R}\left(W_{1, m}\right)$ (by Theorem 7.11). Also, $g_{\bar{T} \cup\left\{c_{0}\right\}}\left(v_{i-1}, v_{i+2}\right)=2=$ $g_{\bar{T} \cup\left\{c_{0}\right\}}\left(v_{i+2}, v_{i+5}\right)$ and hence $\bar{T} \notin \mathcal{R}\left(W_{1, m}\right)$. So, $T \in \hat{\mathcal{R}}\left(W_{1, m}\right)$. So, $l_{\hat{r}}\left(W_{1, m}\right) \leq$ $|T|=4$. Thus, $l_{\hat{r}}\left(W_{1, m}\right)=4$ if $m=7,8$.

When $m \geq 9$, let $S^{\prime}=\left\{v_{i}: 8 \leq i \leq m\right\}$ and $W=\left\{v_{2}, v_{3}, v_{5}, v_{6}\right\}$. Then, $W$ resolves all the pairs $x, y$ whenever either $x, y \in\left\{v_{j}: 1 \leq j \leq 7\right\}$, or $x \in$ $\left\{v_{j}: 1 \leq j \leq 7\right\}$ and $y \in W$. That is, it will not resolve any two vertices in $G^{\prime}=\left\langle S^{\prime}\right\rangle$. But then, as $G^{\prime} \cong F\left(1,\left|S^{\prime}\right|\right)$, we have $T=W \cup W^{\prime} \in \mathcal{R}\left(W_{1, m}\right)$ and 
$\bar{T} \notin \mathcal{R}\left(W_{1, m}\right)$, for every $W^{\prime} \in \mathcal{R}\left(F\left(1,\left|S^{\prime}\right|\right)\right)$. Thus, $l_{\hat{r}}\left(W_{1, m}\right) \leq|T|=|W|+$ $l_{r}\left(F\left(1,\left|S^{\prime}\right|\right)\right)=4+\left\lfloor\frac{2((m-7)+1)}{5}\right\rfloor=\left\lfloor\frac{2(m+4)}{5}\right\rfloor$ (by Theorem 7.4). On the otherhand, From Theorem 7.2 and Lemma 7.19 it follows, for all $m \geq 9$, that $l_{\hat{r}}\left(W_{1, m}\right) \geq$ $l_{r}\left(W_{1, m}\right)+\xi=\left\lfloor\frac{2(m+2)}{5}\right\rfloor+\xi$, where $\xi=2$ if $m \equiv 1(\bmod 5)$ and $\xi=1$ otherwise. This is same as saying $l_{\hat{r}}\left(W_{1, m}\right) \geq\left\lfloor\frac{2(m+4)}{5}\right\rfloor$.

\section{Conclusion}

In this paper, it is shown that there are some graphs whose foul resolving number is constant but its metric dimension is not constant. However, for most of the graphs the powerful resolving number is constant whenever metric dimension is constant. Finally we found, for all the graphs considered here, that the maximal resolving number is not independent of its order. In conclusion, the new dimensions introduced and analyzed here may be a new network parameter in the study of network theory, and the networks with prescribed dimensions is the essence in network security issues.

Acknowledgement. The authors are very much thankful to the Managements and Principals of Dr. Ambedkar Institute of Technology, REVA University, and Nitte Meenakshi Institute of Technology, for their constant support and encouragement during the preparation of this paper. Also special thanks to the anonymous referees for their suggestions for the improvement of this paper.

\section{REFERENCES}

[1] F. Buckley and F. Harary, Distance in graphs, Addison-Wesley, (1990).

[2] J. Caceres, C. Hernando, M. Mora, I.M. Pelayoe, M.L. Puertas, C. Seara, D.R. Wood, On the metric dimension of some families of graphs, Electron. Notes Discrete Math. 22(2005), 129-133.

[3] Gary Chartrand, Linda Eroh, Mark A. Johnson, and Ortrud R. Oellermann, Resolvability in graphs and the metric dimension of a graph, Discrete Appl. Math., 105(1-3)(2000), 99-113.

[4] Hartsfield Gerhard and Ringel, Pearls in graph theory, Academic Press, USA, (1994).

[5] F. Harary and R.A. Melter, On the metric dimension of a graph, Ars Combin., 2(1976), 191-195.

[6] S. Khuller, B. Raghavachari and A. Rosenfied, Landmarks in graphs, Discrete Appl. Math., 70 (1996), 217-229.

[7] V. Saenpholphat and Ping Zhang, Connected resolvability of graphs, Czechoslovak Math. J., 53(128)(4)(2003), 827-840.

[8] A. Sebo and E. Tannier, On metric generators of graphs, Math. Opr. Res., 29(2) (2004), 383-393.

[9] B. Shanmukha, B. Sooryanarayana and K.S. Harinath, Metric dimention of wheels, Far East J. Appl. Math., 8(3) (2002), 217-229.

[10] P. J. Slater, Leaves of trees, In Proc. 6th Southeastern Conf. on Combinatorics, Graph Theory, and Computing, Congr. Numer.,14(1975), 549-559. 
[11] B. Sooryanarayana and Shanmukha B, A note on metric dimension, Far East J. Appl. Math., $\mathbf{5}(3)(2001), 331-339$

[12] B. Sooryanarayana, On the metric dimension of graph, Indian. J. Pure Appl. Math., 29(4)(1998), 413-415.

[13] B. Sooryanarayana and Suma A. S, On classes of neighborhood resolving sets of a graph, Electron. J. Graph Theory Appl., 6(1)(2018), 29-36.

[14] B. Sooryanarayana, Shreedhar K and Narahari N, Metric dimension of generalized wheels, Arab J. Math. Sci. 25(2)(2019), 131-144. 